\title{
Development of Amplifier with Pulse Shaper for High Rate MWPC
}

\author{
I. Kamiji*, N. Sasao, T. Nomura, H. Nanjo, T. Masuda, N. Kawasaki, D. Naito, Y. \\ Maeda, S. Seki, K. Nakagiri, S. Shinohara \\ Kyoto University \\ E-mail: i.kamijiescphys.kyoto-u.ac.jp
}

\begin{abstract}
An amplifier has been developed for wire chambers used as an in-beam detector for charged particles in the KOTO experiment, which aims at discovering a CP-violating rare decay of long-lived neutral $\mathrm{K}$ meson, $K_{L} \rightarrow \pi^{0} v \bar{v}$. Due to in-beam condition of the wire chambers, the maximum counting rate is estimated to reach $700 \mathrm{kHz}$ per channel. Because the output signals of the wire chambers have a long tail of low frequency components coming from slow drift of ions which remains tens of microseconds, pulse pile-up and baseline fluctuation are expected in such a high rate operation. In order to suppress these undesirable phenomena, a pulse shaping circuit was integrated into the signal amplifier for the wire chamber, which consists of three pole-zero cancellation networks. The performance of the pulse shaping circuit was confirmed by using a prototype single-channel MWPC. The achieved pulse width was $40 \%$ of the original one in FWHM. The total pulse width was $150 \mathrm{~ns}$, where any long tail exceeding the total width vanished.
\end{abstract}

Flavor Physics \& CP Violation 2015,

May 25-29, 2015

Nagoya, Japan

\footnotetext{
* Speaker.
} 


\section{Introduction}

\subsection{The KOTO Experiment}

The KOTO experiment aims to discover a $\mathrm{CP}$-violating rare decay of the long-lived neutral $\mathrm{K}$ meson, $K_{L} \rightarrow \pi^{0} v \bar{v}$. The branching ratio has been calculated as $2.4 \times 10^{-11}$ with a small theoretical uncertainty (2\%) [1] in the Standard Model (SM). This theoretical cleanness makes the branching ratio sensitive to the contribution of new physics beyond the SM. Figure 1 shows a cross-sectional view of the detector system of the KOTO experiment. For the detection of $K_{L} \rightarrow \pi^{0} v \bar{v}$, two photons are measured with an electromagnetic calorimeter made of undoped cesium iodide crystals (CsI), and the other detectors ensure no extra visible particle exists.

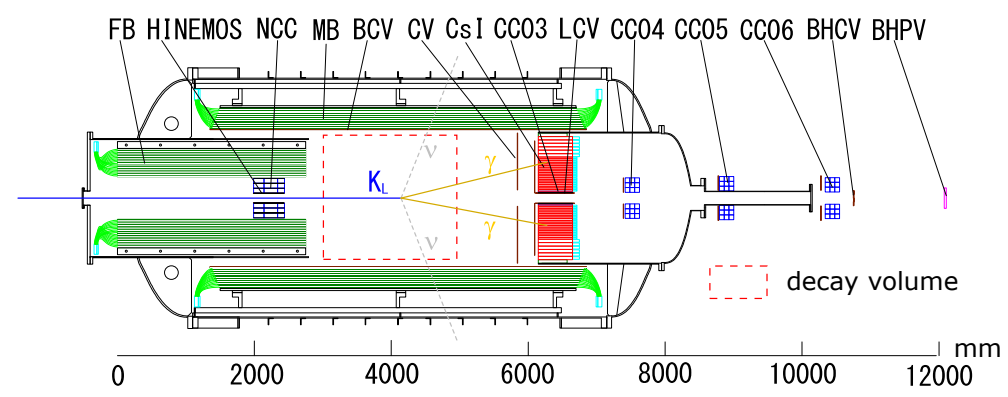

Figure 1: Detector system of the KOTO experiment. A typical $K_{L} \rightarrow \pi^{0} v \bar{v}$ decay is also shown.

\subsection{Beam Hole Charged Veto (BHCV)}

Beam Hole Charged Veto (BHCV) is an in-beam detector for charged particles passing through the beam hole of the calorimeter. It consists of three successive wire chambers. The configuration of the wire chamber is shown in Fig. 2 and Table 11. Because BHCV is directly exposed to the beam, its counting rate reaches $700 \mathrm{kHz}$ per channel. Output signals of wire chambers, however, have a long tail of low frequency components lasting tens of microseconds due to slow drift of ions. Therefore such a high rate operation causes frequent pulse overlapping and baseline shift, which lead to loss of energy and timing information. To cope with these problems, a pulse shaping circuit has been developed for the amplifier of BHCV described in this paper, which reduces low frequency components to make waveforms shorter.

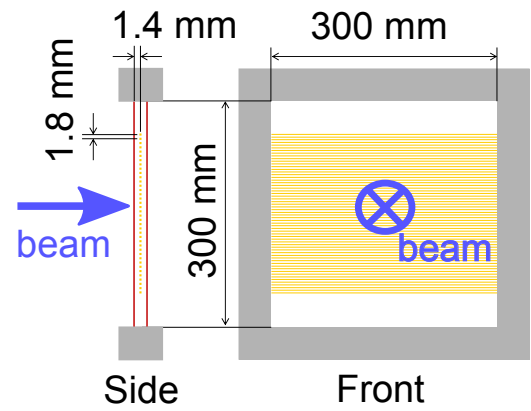

Figure 2: Wire chamber for BHCV.

\begin{tabular}{cc}
\hline wire diameter / length & $50 \mu \mathrm{m} / 30 \mathrm{~cm}$ \\
number of wires & $160(10$ wires $\times 16$ channels $)$ \\
wire-wire spacing & $1.8 \mathrm{~mm}$ \\
wire-cathode gap & $1.4 \mathrm{~mm}$ \\
cathode plane & graphite-coated kapton film \\
gas & $50-\mu \mathrm{m}$ thick \\
operation voltage & $\mathrm{CF}_{4}: \mathrm{n}-$ pentane $=55: 45$ \\
capacitance per channel & $2.7 \mathrm{kV}$ to $2.9 \mathrm{kV}$ \\
\hline
\end{tabular}

Table 1: Specification of the wire chamber. 


\section{Desgin of Amplifier}

\subsection{Schematic Diagram}

Figure 3 is a schematic diagram of the amplifier for BHCV. It consists of two parts: preamplifying part and pulse shaping part. The preamplifying part has a charge amplifier with a time constant of $6 \mathrm{~ns}$. The pulse shaping part is a series of three successive pole-zero cancellation networks (right-top side of Fig. 3).

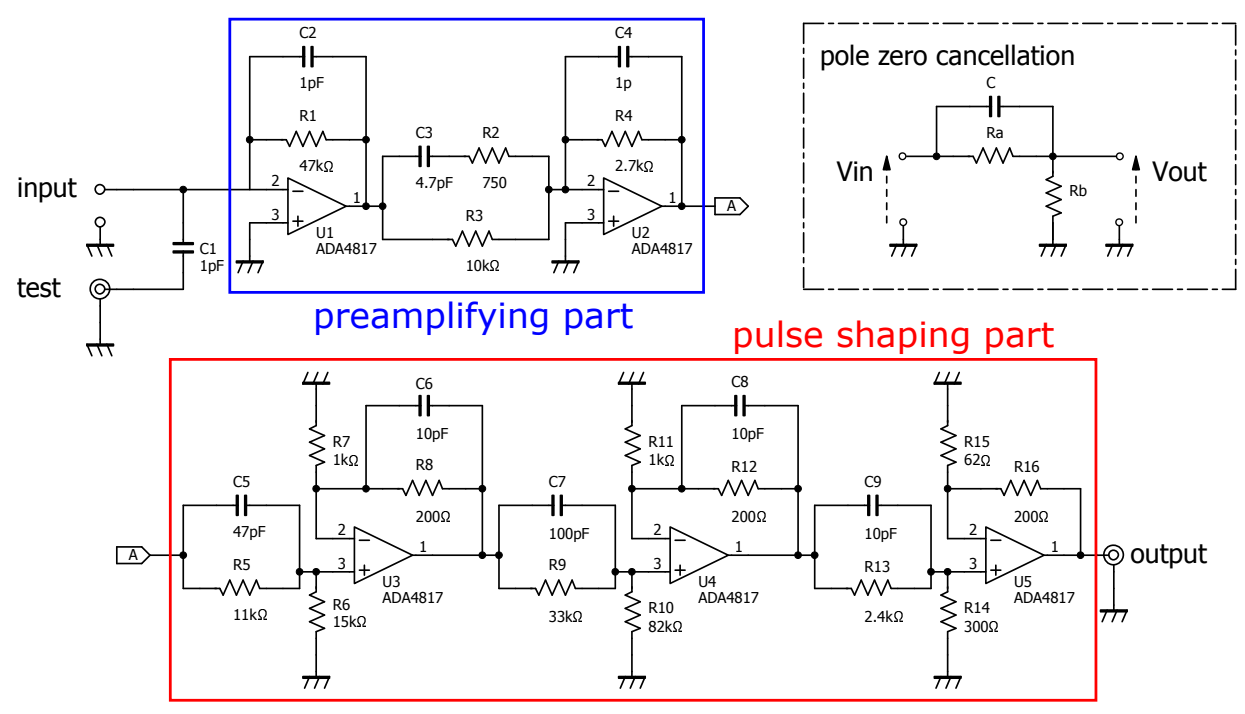

Figure 3: Schematic diagram of the amplifier for BHCV. The schematic of the pole-zero cancellation network used in the pulse shaping part is extracted to the right-top side.

\subsection{Principle of Pulse Shaping}

To determine the circuit parameters of the pulse shaping part, signal waveforms at the output of the preamplifying part were collected, using a single channel wire chamber ${ }^{1}$ with ${ }^{90} \mathrm{Sr}$. Figure 4 shows a waveform template of output signals. The tail region of the waveform can be described well by using the following function:

$$
A \exp \left(-t / \tau_{a}\right)+B \exp \left(-t / \tau_{b}\right)+C \exp \left(-t / \tau_{c}\right)
$$

where all the fitted parameters are also shown in Fig. 4 This function can be reduced to a single exponential with a time constant of $\tau_{a}$ by using the first and second pole-zero cancellations [2].

After passing those two pole-zero cancellations, the signal waveform becomes shorter without remaining long tail as shown in Fig. 5, This waveform is fitted well by the following function ${ }^{2}$ :

$$
D\left(t-t_{0}\right) \exp \left(-\left(t-t_{0}\right) / \tau_{d}\right)
$$

The fitting result is also shown in Fig. 5. The last pole-zero cancellation removes the multiplication by $\left(t-t_{0}\right)$ component. So the resultant output of the pulse shaping part is an exponential waveform with a time constant of $24 \mathrm{~ns}$.

\footnotetext{
${ }^{1}$ It has the same wire diameter, wire-wire spacing, and wire-cathode gap as those in BHCV.

${ }^{2}$ The reason why this function is not a single exponential is that Eq. 2.1 is just an approximation only valid in the tail region of the waveform in Fig. 4
} 


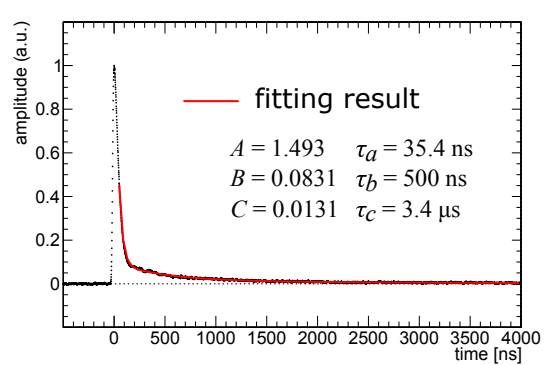

Figure 4: Waveform of preamplifying part with a sin- Figure 5: Waveform before the last pole-zero cancelgle channel wire chamber.

lation.

\section{Performance of Pulse Shaping Circuit}

The performance of the pulse shaping circuit was checked by a prototype amplifier (Fig. 6). Figure 7 shows a comparison of signal waveforms before (blue) and after (red) the pulse shaping. There is no remaining long tail after the shaping and the FWHM of the output waveform became 26 ns, which was $40 \%$ of that of the waveform before the shaping. The time constant of the resultant exponential tail was $24 \mathrm{~ns}$, and its total with was $150 \mathrm{~ns}$.

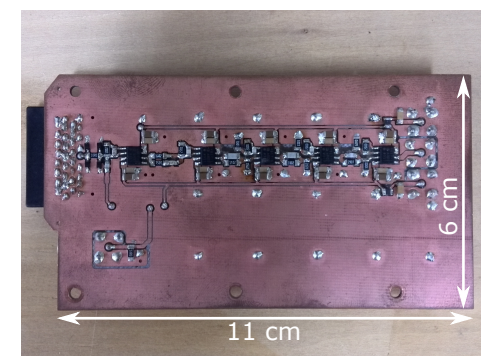

Figure 6: Prototype amplifier.

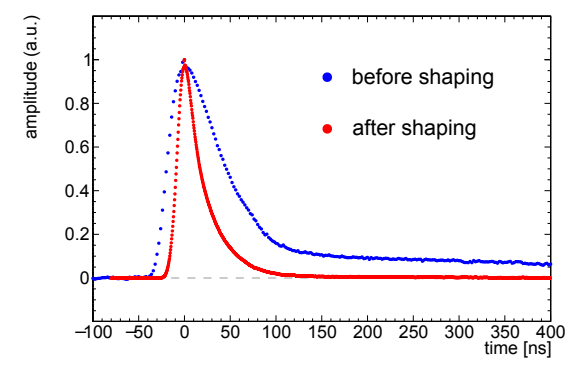

Figure 7: Comparison of waveforms. The waveform before shaping is the same as shown in Fig. 4

\section{Conclusion}

An amplifier with pulse shaping circuit was developed for the wire chambers used in high rate environment at the in-beam region of the KOTO experiment. The shaping circuit consisted of three pole-zero cancellation networks. The performance of the pulse shaping circuit was tested by using a prototype amplifier and a single channel wire chamber. It reduced a long tail in the output signals of the wire chamber, and the achieved pulse width was $40 \%$ of the original one in FWHM, and its total width was $150 \mathrm{~ns}$.

\section{References}

[1] J. Brod, M. Gorbahn, E. Stamou, Two-loop electroweak corrections for the $K \rightarrow \pi v \bar{v}$ decays, phys. rev. D 83 (2011) 034030.

[2] R. A. Boie, A. T. Hrisoho, P. Rehak, Signal Shaping and Tail Cancellation for Gas Proportional Detectors at High Counting Rates, IEEE Trans. Nuc. Sci. NS-28, No.1 (1981) 603 